\title{
Video Article \\ Exon Skipping in Directly Reprogrammed Myotubes Obtained from Human Urine-Derived Cells
}

\author{
Hotake Takizawa ${ }^{1}$, Mitsuto Sato ${ }^{1}$, Yoshitsugu Aoki ${ }^{1}$ \\ ${ }^{1}$ Department of Molecular Therapy, National Institute of Neuroscience, National Center of Neurology and Psychiatry \\ Correspondence to: Yoshitsugu Aoki at tsugu56@ncnp.go.jp \\ URL: https://www.jove.com/video/60840 \\ DOI: doi: $10.3791 / 60840$
}

Keywords: Genetics, Issue 159, Duchenne muscular dystrophy (DMD), urine-derived cells, exon skipping, precision medicine, skeletal muscle, clinical trial

\section{Date Published: 5/7/2020}

Citation: Takizawa, H., Sato, M., Aoki, Y. Exon Skipping in Directly Reprogrammed Myotubes Obtained from Human Urine-Derived Cells. J. Vis. Exp. (159), e60840, doi:10.3791/60840 (2020).

\section{Abstract}

Duchenne muscular dystrophy (DMD), a progressive and fatal muscle disease, is caused by mutations in the $D M D$ gene that result in the absence of dystrophin protein. To date, we have completed an investigator-initiated first-in-human study at the National Center of Neurology and Psychiatry based on the systemic injection of the morpholino oligonucleotides which are prone to exon-53 skipping. For the effective treatment of DMD, in vitro testing with myoblasts derived from DMD patients to screen drugs and assess patient eligibility before undertaking clinical trials is thought to be essential. Very recently, we reported a new MYOD1-converted urine-derived cell (UDC) treated with the histone methyltransferase inhibitor (3-deazaneplanocin A hydrochloride), as a cellular model of DMD. The new autologous UDC might show phenocopy of the diseasespecific phenotypes of DMD, leading to the application of precision medicine in a variety of muscle-related diseases. In this article, we describe a detailed protocol for efficient modelling of DMD muscle cells using MYOD1-converted UDCs along with reverse transcriptase polymerase chain reaction (RT-PCR), Western blotting, and immunocytochemistry to evaluate the restoration of dystrophin mRNA and protein levels after exon skipping.

\section{Video Link}

The video component of this article can be found at https://www.jove.com/video/60840/

\section{Introduction}

Duchenne muscular dystrophy (DMD), a progressive, fatal muscle disease, is caused by frame-shift mutations in the $D M D$ gene that result in the absence of dystrophin protein ${ }^{1}$. Antisense oligonucleotide-based exon skipping therapy is thought to be promising for DMD. This therapy is based on the conversion of the severer DMD phenotype to the milder Becker muscular dystrophy-like phenotype by altering pre-mRNA splicing to restore the $D M D$ reading frame ${ }^{2}$. We have recently completed a first-in-human study based on repeated intravenous administration of the phosphorodiamidate morpholino oligomer (PMO) viltolarsen, which can induce exon 53 skipping in DMD, and demonstrated an excellent safety profile, promising efficacy, and acceptable pharmacokinetic parameters (registered as UMIN: 000010964 and ClinicalTrials.gov: NCT02081625) ${ }^{3}$.

However, to develop cost-effective and efficient treatments for the disease, in vitro tests using primary muscle cells obtained from DMD patients are essential for drug screening and patient eligibility verification before undertaking clinical trials, as well as biomarkers that reflect the efficacy of exon skipping therapies during human trials ${ }^{4}$. Very recently, we reported a novel technology to develop patient-specific MYOD1-converted urine-derived cells (UDCs) ${ }^{5,6}$ as a primary myoblast model of $\mathrm{DMD}^{7}$. Thus, to generate the myoblasts, only the collection of urine from patients is required and no invasive procedure is needed. In this article, we describe a detailed protocol for efficient modelling of DMD muscle using MYOD1-converted UDCs treated with 3-deazaneplanocin A hydrochloride to evaluate the restored dystrophin mRNA and protein after exon skipping.

\section{Protocol}

The Ethics Committee of the National Center of Neurology and Psychiatry approved this study (approval ID: A2017-018, A2018-029). All individuals gave informed consent before providing urine. All experiments were performed under the relevant guidelines and regulations.

\section{Isolation and primary culture of UDCs}

NOTE: UDCs were isolated according to a previously published protocol ${ }^{8,9,10}$ with some modifications.

1. Collect urine samples during spontaneous micturition in sterilized plastic bottles. 
NOTE: Sterilization of the external urethral orifice is not needed. Midstream urine is desirable to reduce the risk of viral contamination. If the stock time before the next procedure is $>1 \mathrm{~h}$, urine samples should be transferred to $4{ }^{\circ} \mathrm{C}$ to preserve the cell viability. However, a temperature of $<4{ }^{\circ} \mathrm{C}$ should be avoided because insoluble precipitates may appear.

2. Centrifuge the entire urine sample at $400 \times g$ for $10 \mathrm{~min}$ at room temperature.

3. Aspirate the supernatant, leaving $1 \mathrm{~mL}$ in the tube.

4. Resuspend the pellets individually in the remaining $1 \mathrm{~mL}$ of urine and then collect those in a single $50 \mathrm{~mL}$ tube.

5. Add $10 \mathrm{~mL}$ of washing buffer consisting of $99 \mathrm{~mL}$ of PBS without calcium and magnesium, $1 \%$ penicillin/streptomycin (P/S), $0.5 \mu \mathrm{g} / \mathrm{mL}$ amphotericin $B$, and centrifuge the samples at $200 \times g$ for $10 \mathrm{~min}$ at room temperature.

6. Aspirate the supernatant, leaving $0.2 \mathrm{~mL}$ in the tube.

7. Resuspend the cell pellets in $4.5 \mathrm{~mL}$ of primary medium composed of a 1:1 mixture of high glucose Dulbecco's modified Eagle medium (DMEM)without sodium pyruvate and Ham's F-12 nutrient mix supplemented with recombinant human epidermal growth factor (EGF), insulin, hydrocortisone, epinephrine, T3, transferrin, 10\% tetracycline-free fetal bovine serum (FBS), $1 \% \mathrm{P} / \mathrm{S}$, and $0.5 \mu \mathrm{g} / \mathrm{mL}$ amphotericin B.

8. Seed the cells in three wells of gelatine-coated six well plates (total volume of each well, $1.5 \mathrm{~mL}$ ). Culture humidified at $37{ }^{\circ} \mathrm{C}$ and $5 \% \mathrm{CO}_{2}$ for $24 \mathrm{~h}$.

9. Add $1.5 \mathrm{~mL}$ of the primary medium daily for the next 3 days.

10. On day 4, replace the medium with $1.5 \mathrm{~mL}$ of growth medium supplemented with recombinant human EGF, insulin, hydrocortisone, epinephrine, T3, transferrin, $15 \%$ tetracycline-free FBS, $0.5 \%$ L-alanine-L-glutamine, $0.5 \%$ nonessential amino acids, and $2.5 \mathrm{ng} / \mathrm{mL}$ fibroblast growth factor-basic (bFGF), recombinant human platelet-derived growth factor (PDGF), EGF, and 1\% P/S.

11. Change the growth medium every other day. NOTE: UDC colonies appear within a week.

12. When the UDC culture becomes $80-90 \%$ confluent, remove the medium and wash cells with PBS, split all the cells using $0.25 \%$ trypsinEDTA and seed at $3,000-5,000$ cells $/ \mathrm{cm}^{2}$ onto a new gelatine-coated $60 \mathrm{~mm}$ dish (passage 1).

NOTE: The UDCs can be stored in liquid nitrogen. UDCs are usually divided at $60-70 \%$ confluency in $60 \mathrm{~mm}$ culture dish into three stock tubes.

\section{Retroviral construct}

1. Amplify the coding region of MYOD1 (NM_002478.4) plasmid by polymerase chain reaction (PCR). NOTE: The mixture for MYOD1 amplification and conditions for the thermal cycler are shown in Table 1 and Table 2, respectively.

2. Detect a single band of about 1,000 bp size by $0.7 \%$ agarose gel electrophoresis using $1 \mu \mathrm{L}$ of the amplified PCR product to confirm that the MYOD1 sequence is amplified successfully.

3. Clean the PCR product using the clean-up kit and determine its concentration with a spectrophotometer.

4. Incubate the mixture as shown in Table 3 at $37^{\circ} \mathrm{C}$ overnight to digest retroviral vector with a Tet-on system and puromycin resistant gene at restriction enzyme-targeted regions in the multiple cloning site.

5. Detect a single band by $0.7 \%$ agarose gel electrophoresis using $1 \mu \mathrm{L}$ of the digested product to confirm that the retroviral vector was digested successfully.

6. Clean the digested product using clean-up kit and determine its concentration by spectrophotometer.

7. To clone the amplified MYOD1 fragment (produced by steps 2.1-2.3) into the digested retroviral vector (produced by steps 2.4-2.6), perform an in-fusion cloning reaction. Set up the reaction as shown in Table 4, incubate the reaction for 15 min at $50^{\circ} \mathrm{C}$, and then place on ice.

8. Perform transformation using $E$. coli competent cells according to the manufacturer's instructions (Table of Materials).

9. Select the transformed competent cells by culturing on an LB culture plate consisting of $10 \mathrm{~g} / \mathrm{L}$ bacto tryptone, $5 \mathrm{~g} / \mathrm{L}$ bacto yeast extract, $5 \mathrm{~g} / \mathrm{L}$ $\mathrm{NaCl}, 15 \mathrm{~g} / \mathrm{L}$ bacto agar, and $50 \mathrm{mg} / \mathrm{L}$ ampicillin.

10. Pick up the selected colony and culture in LB culture medium without bacto agar at $200 \mathrm{rpm}$ at $37^{\circ} \mathrm{C}$ overnight.

11. Purify the MYOD1-inserted retroviral vectors using a plasmid purification kit (Table of Materials) and quantify using a spectrophotometer.

12. Confirm that $M Y O D 1$ is correctly inserted into the retroviral vector by direct sequencing of the PCR product amplified by the forward and reverse primers targeted respectively on both sides across the inserted MYOD1 sequence.

NOTE: The MYOD1 sequence can be detected sandwiched between retroviral vector sequences when in-fusion cloning is successful.

13. For retroviral production, seed the packaging cells at 50,000 cells $/ \mathrm{cm}^{2}$ on $10 \mathrm{~cm}$ collagen-coated plates and culture in DMEM with $10 \%$ FBS humidified at $37^{\circ} \mathrm{C}$ and $5 \% \mathrm{CO}_{2}$ for $24 \mathrm{~h}$.

14. When the packaging cells proliferate to $80 \%$ confluency, mix $30 \mu \mathrm{g}$ of MYOD1-inserted retroviral vectors, $30 \mu \mathrm{g}$ of packaging vectors, and transfection reagent containing cell-penetrating peptide (Table of Materials) by vortexing, and incubate them for $10 \mathrm{~min}$.

15. Add the incubated mixture to the medium of packaging cells and culture humidified at $37{ }^{\circ} \mathrm{C}$ and $5 \% \mathrm{CO}_{2}$. NOTE: Collagen coating is necessary. Transfection may be better at $24 \mathrm{~h}$ or more after seeding because packaging cells easily detach from the culture plate.

16. After $4 \mathrm{~h}$ or overnight, change the medium to fresh growth medium.

17. Collect the viral supernatant and replace with fresh medium at 24 and $48 \mathrm{~h}$ after cotransfection and combine the supernatant.

18. To concentrate the viral supernatant, mix it with the concentrator reagent and incubate at $37^{\circ} \mathrm{C}$ overnight, then centrifuge at $1,500 \times g$ for 45 $\min$ at $4{ }^{\circ} \mathrm{C}$.

19. Filter the retrovirus supernatant through a PVDF filter with $0.45 \mu \mathrm{m}$ pores.

20. Check the titer of the retroviral vector using a quantitative PCR kit and thermal cycler system according to the manufacturer's instructions.

21. Divide the viral supernatant into small aliquots and stock them at $-80{ }^{\circ} \mathrm{C}$.

\section{Infection with MYOD1-retroviral vector in UDCs}

1. Seed the UDCs at $3,000-5,000$ cells $/ \mathrm{cm}^{2}$ on a gelatine-coated $60 \mathrm{~mm}$ dish.

2. After $24 \mathrm{~h}$ of seeding, infect the thawed retrovirus (step 2.21) at a multiplicity of infection of 200 by adding hexadimethrine bromide at a concentration of $8 \mu \mathrm{g} / \mathrm{mL}$. 
3. After a $24 \mathrm{~h}$ incubation humidified at $37^{\circ} \mathrm{C}$ and $5 \% \mathrm{CO}_{2}$, replace the culture medium with fresh growth medium containing $1 \mu \mathrm{g} / \mathrm{mL}$ puromycin to select the MYOD1-transduced cells. Change the medium every other day.

NOTE: When selecting for MYOD1-positive cells, $1 \mu \mathrm{g} / \mathrm{mL}$ puromycin is usually used for selection. The appropriate dose should be determined. Use a plate containing untransfected cells and choose the dose that kills all the cells in 3-5 days. MYOD1-positive cells should be selected within 7-10 days after adding puromycin. The MYOD1-transduced UDCs can be stored in liquid nitrogen.

\section{Myogenic differentiation of MYOD1-transduced UDCs treated with 3-deazaneplanocin A hydrochloride (DZNep)}

NOTE: Recently, it has been reported that DZNep, a histone methyltransferase inhibitor, could significantly promote the expression of MYOGENIN, one of the late muscle regulatory factors, and also lead to myotube differentiation ${ }^{7}$.

1. Plate MYOD1-transduced UDCs in the collagen-coated wells at a density of $3.5 \times 10^{4} \mathrm{cells} / \mathrm{cm}^{2}$. Culture humidified at $37{ }^{\circ} \mathrm{C}$ and $5 \% \mathrm{CO}$.

2. After $24 \mathrm{~h}$, change the growth medium to differentiation medium composed of high glucose DMEM with L-alanine-L-glutamine, $5 \%$ horse serum, ITS supplement, $1 \mu \mathrm{g} / \mathrm{mL}$ doxycycline, and $5 \mu \mathrm{M}$ DZNep.

NOTE: The $10 \mathrm{mM}$ DZNep solution can be stored at $-80^{\circ} \mathrm{C}$ for 3 months. Use a defrost freezer and avoid repeated freeze-thaw cycles. Both MYOD1 activated by doxycycline and DZNep suppress proliferation and promote the myogenic differentiation of UDCs. Therefore, it is recommended that doxycycline and DZNep are added after the induction of myogenic differentiation. DZNep promotes myogenic differentiation of the MYOD1-UDCs in a dose-dependent manner. On the other hand, it shows cytotoxicity at a high concentration. Therefore, determine the appropriate concentration of DZNep, ranging from 1-10 $\mu \mathrm{M}$ depending on its effects on myogenic differentiation and cellular bioavailability.

3. After 3 days, change the differentiation medium to fresh differentiation medium without DZNep. Then, change the medium every 3 days. NOTE: UDCs fuse to each other and form myotubes within 1-2 weeks after differentiation.

\section{Exon skipping in MYOD1-converted UDCs}

NOTE: Here, three protocols are described to evaluate exon skipping in patient-derived cells: 1) reverse transcriptase polymerase chain reaction (RT-PCR) of dystrophin mRNA; 2) semiquantification of restored dystrophin protein signal by Western blot; and 3) semiquantification of restored dystrophin fluorescence signal by immunocytochemistry. All the methods can detect exon skipping in a dose-dependent manner.

1. Evaluation of exon skipping efficiency by RT-PCR

1. To transfect antisense oligonucleotide (ASO) into MYOD1-converted UDCs obtained from DMD patients on the day 7 after differentiation, mix ASO, transfection reagent (Table of Materials), and differentiation medium to a final concentration of 1-10 $\mu \mathrm{M}$. Culture humidified at $37{ }^{\circ} \mathrm{C}$ and $5 \% \mathrm{CO}_{2}$.

2. After $72 \mathrm{~h}$ of incubation with $\mathrm{ASO}$, change the medium to fresh differentiation medium without ASO.

3. From 3-7 days after ASO transfection, remove differentiation medium and wash $1 \mathrm{x}$ with PBS. Add cell lysis buffer, lyse the UDCs and harvest the total RNA using an RNA extraction kit.

4. Measure the RNA concentration with a spectrophotometer.

5. Combine the required reagents for one-step RT-PCR reaction in PCR tubes according to Table $\mathbf{5}$.

6. Place the PCR tubes with the mixture in a thermocycler. Run the thermocycler, according to Table 6.

7. Perform microchip electrophoresis and calculate the exon skipping efficiency using the molar concentration as below. Exon skipping efficiency $(\%)=$ skipped band / (skipped band + non-skipped band) $\times 100$ NOTE: Store the PCR product in a refrigerator at $4{ }^{\circ} \mathrm{C}$ for short-term storage or $-20{ }^{\circ} \mathrm{C}$ for long-term storage.

2. Detection of dystrophin after exon skipping by Western blotting

1. Transfect ASO and culture MYOD1-UDCs according to steps 5.1.1 and 5.1.2.

2. Change the medium every 3 days.

3. After 2 weeks of differentiation, extract the total protein from the cultured cells using radioimmunoprecipitation assay (RIPA) buffer containing protease inhibitors.

4. Sonicate the lysates on ice and centrifuge at $14,000 \times g$ for 15 min at $4{ }^{\circ} \mathrm{C}$.

5. Collect the supernatant and determine protein concentrations using a BCA protein assay kit.

6. Add $15 \mu \mathrm{g}$ of total protein in a $0.5 \mathrm{~mL}$ tube and dilute by adding the RIPA buffer containing protease inhibitors to a total volume of 10 $\mu \mathrm{L}$. Run $15 \mu \mathrm{g}$ of total protein per lane.

7. Add sample buffer, reducing agent, and deionized water as shown in Table 7. Denature the cell lysates at $70{ }^{\circ} \mathrm{C}$ for $10 \mathrm{~min}$

8. Prepare the tris-acetate running buffer containing $8.95 \mathrm{~g} / \mathrm{L}$ tricine, $6.06 \mathrm{~g} / \mathrm{L}$ tris base, $1.0 \mathrm{~g} / \mathrm{L}$ sodium dodecyl sulfate (SDS).

9. Load the sample $(20 \mu \mathrm{L})$ onto tris-acetate $3-8 \%$ gel and perform electrophoresis at $150 \mathrm{~V}$ for $75 \mathrm{~min}$.

10. Prepare the blotting buffer without methanol.

11. Soak the PVDF membrane for $20 \mathrm{~s}$ in methanol and then in the blotting buffer until use (at least $10 \mathrm{~min}$ ). Cut the PVDF membrane to a size of $6 \times 8 \mathrm{~cm}$ using mini gels and $8 \times 12 \mathrm{~cm}$ using midi gels.

12. Cut the blotting papers to the same size as that of the PVDF membrane and soak those in the blotting buffer until use.

13. After electrophoresis, cut the gel to the same size as that of the PVDF membrane and soak the gel in distilled water.

14. Place the blotting papers, PVDF membrane, and gel on the semidry transfer apparatus (Figure 1). Transfer at $4 \mathrm{~mA} / \mathrm{cm}^{2}$ for $30 \mathrm{~min}$.

15. Rinse the membrane $2 x$ with distilled water.

16. Prepare anti-dystrophin (1:500) and anti- $\alpha$-tubulin $(1: 1,200)$ antibody as the primary antibodies.

17. Prepare HRP-conjugated anti-mouse antibody $(1: 100)$ as a secondary antibody.

18. Incubate the membranes with the primary antibodies, wash with wash buffer, then incubate with the secondary antibody using an automated Western-processing device (Table of Materials) at room temperature. 
NOTE: Anti- $\alpha$-tubulin antibody is usually used as a loading control. The mixed primary antibody solutions, including 1:500 antidystrophin and 1:1,200 anti- $\alpha$-tubulin antibodies work well when the antibody reaction is performed simultaneously.

19. Rinse the membrane in distilled water.

20. Detect the proteins using chemiluminescent detection reagent and a charge-coupled device (CCD) camera-based imager.

21. Analyse the data using appropriate software.

3. Detection of dystrophin after exon skipping by immunocytochemistry

1. Directly reprogram the UDCs into the myotubes in collagen-coated 96 well plate according to steps 4.1-4.3.

2. Transfect the ASO and culture MYOD1-UDCs according to steps 5.1.2 and 5.1.3.

3. After 2 weeks of differentiation, wash the cells with PBS and fix them in $4 \%$ paraformaldehyde for 10 min at $4{ }^{\circ} \mathrm{C}$.

4. Permeabilize MYOD1-UDCs in $0.1 \%$ nonionic detergent for 10 min at room temperature and block those with $10 \%$ goat serum for 15 $\min$ at $37^{\circ} \mathrm{C}$.

5. Incubate the cells with a primary antibody overnight at $4{ }^{\circ} \mathrm{C}$.

6. Wash the cells with PBS and incubate those with the secondary antibody for $30 \mathrm{~min}$ at room temperature.

NOTE: Here, mouse anti-dystrophin (1:30) is used as the primary antibody, anti-mouse IgG is used as the secondary antibody, and Hoechst $(1: 10,000)$ is used for nuclei staining.

7. Image the plates using a fluorescent microscope and use an analyzer to semiquantify the fluorescence signal automatically in every well under the same condition.

\section{Representative Results}

We could collect the UDCs easily and non-invasively. UDCs formed colonies within a week after starting primary cell culture we observed a marked proliferative ability. The culture of UDCs was straightforward, and bacterial or fungal contamination was rare when the procedure was performed correctly.

Figure 2 shows representative phase-contrast images of the UDC colony a week after primary culture (Figure 2A) and MYOD1-UDCs a week after differentiation (Figure 2B). Figure 3 shows the successful detection of exon skipping in UDCs obtained from DMD patients by RT-PCR. Figure 3A shows RT-PCR analysis of dystrophin after antisense oligonucleotide treatment in DZNep-treated MYOD1-UDCs derived from a 6year-old male with an exon $45-54$ deletion in the $D M D$ gene. The open reading frame was restored by exon 44 skipping. On day 14 following differentiation, we confirmed the induction of exon skipping in a dose-dependent manner (Figure 3B). The upper bands denote native products, and the lower bands denote exon 44-skipped products that restored the open reading frame.

Figure 4 shows the successful detection of dystrophin after exon skipping in the UDCs obtained from DMD patients by Western blotting in a dose-dependent manner. We also detected the restored dystrophin expression using immunocytochemistry (Figure 5). We measured the intensities of dystrophin with a fluorescent microscope 1 week after the antisense oligonucleotide (ASO) transfection on a 96 well plate (Figure 5A). Markedly higher fluorescent signals were observed in MYOD1-UDCs treated with ASO than in MYOD1-UDCs treated with control ASO (Figure 5B).

These results suggest that our new assay can evaluate exon skipping efficiently in MYOD1-UDCs obtained from DMD patients at the mRNA and protein level.

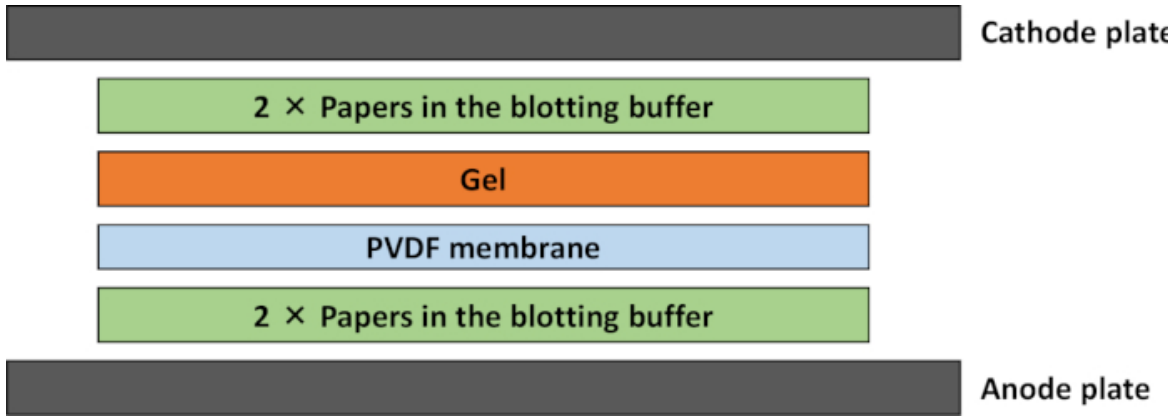

Figure 1: Schematic representation of the transfer stack for semidry Western blot. Two papers soaked in the blotting buffer were laid down at the negative terminal, and two papers soaked in the buffer were stacked on top of this. The gel, which has been soaked in the buffer, was laid gently over the PVDF membrane. Please click here to view a larger version of this figure. 
A

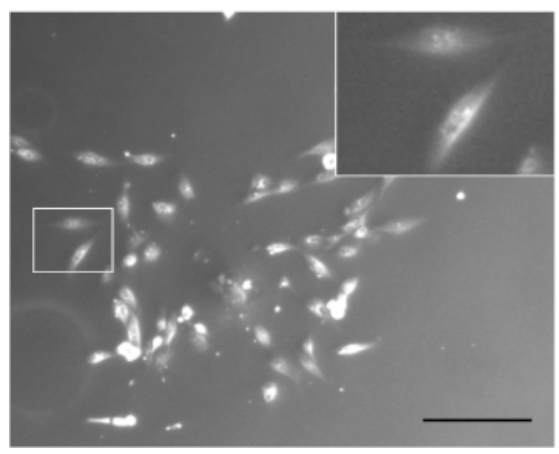

B

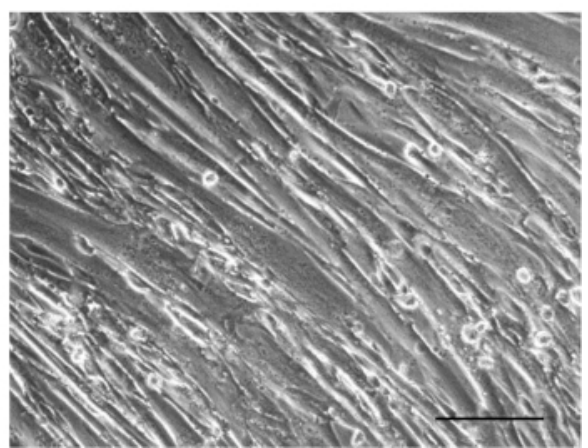

Figure 2: Representative images of the UDCs. (A) Phase-contrast image of UDCs a week after primary culture. Scale bar $=200 \mu \mathrm{m}$. Inset: A magnified image of the area in the white rectangle. (B) Phase-contrast image of MYOD1-UDCs a week after differentiation. Scale bar $=50 \mu \mathrm{m}$. This figure has been modified from Takizawa et al. ${ }^{7}$. Please click here to view a larger version of this figure.

A



B

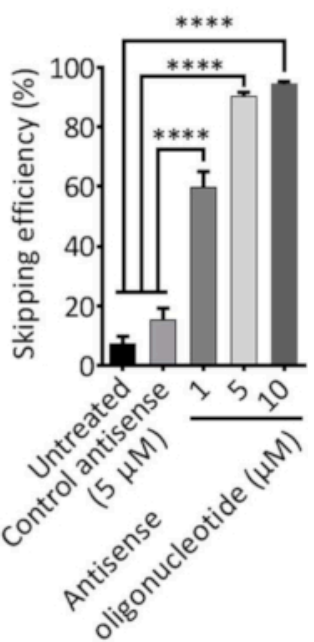

Figure 3: Successful evaluation of exon skipping in urine-derived cells (UDCs) obtained from DMD patients by RT-PCR. (A) RT-PCR analysis of dystrophin after antisense oligonucleotide treatment in 3-deazaneplanocin A hydrochloride (DZNep)-treated MYOD1-UDCs derived from Duchenne muscular dystrophy (DMD) patient with an exon 45-54 deletion. DZNep-treated MYOD1-UDCs were also treated with the control antisense at $1-10 \mu \mathrm{M}$ concentration as controls. The upper bands were unskipped products (Ex 45-54 deletion) that remained out of the reading frame. The lower bands were the exon 44-skipped products (Ex 44-54 deletion and Ex 44 skipped) that restored the open reading frame. (B) Skipping efficiency was calculated as (exon 44-skipped transcript molarity)/(native + exon 44-skipped transcript molarity [marked with arrows]) x $100 \%$ using a microchip electrophoresis system. One-way ANOVA followed by Bonferroni's post hoc test was used to compare the skipping efficiencies $\left(n=3\right.$ for each group, $\left.{ }^{* * *} P<0.0001\right)$. The data are expressed as the mean \pm SEM. This figure has been modified from Takizawa et al. $^{7}$. Please click here to view a larger version of this figure. 
A

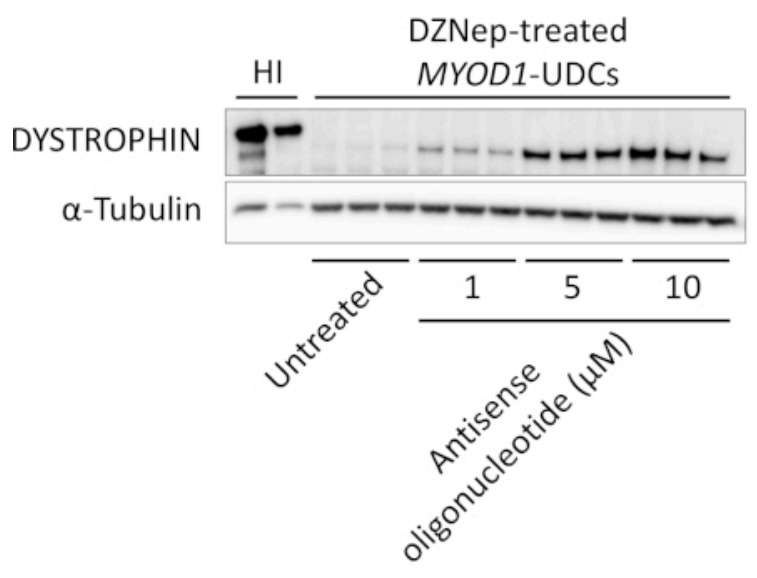

B

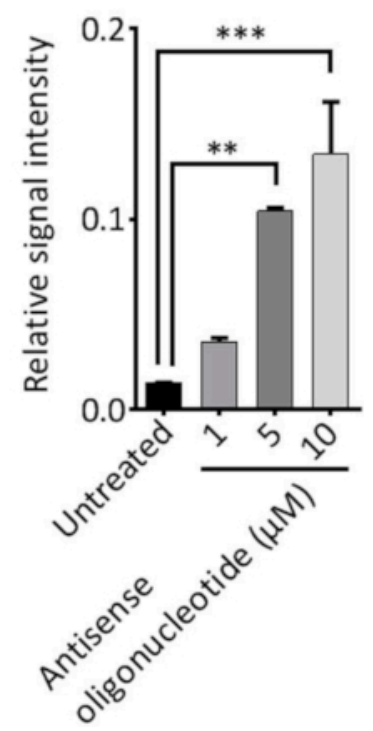

Figure 4: Successful evaluation of exon skipping in urine-derived cells (UDCs) from DMD patients by Western blot. (A) Representative Western blot for dystrophin in DZNep-treated MYOD1-UDCs from DMD patient with an exon 45-54 deletion after exon 44 skipping. For dystrophin detection, anti-dystrophin (against C-terminal) was used. (B) The relative intensities of the bands normalized to a-tubulin expression were compared in patient-derived cells with and without antisense oligonucleotide treatment by performing one-way ANOVA followed by Bonferroni's post hoc test $\left(n=3\right.$ for each group, ${ }^{* *} \mathrm{P}<0.01$, ${ }^{* \star *} \mathrm{P}<0.001, \mathrm{HI}=$ healthy individual). This figure has been modified from Takizawa et al. $^{7}$. Please click here to view a larger version of this figure.

A

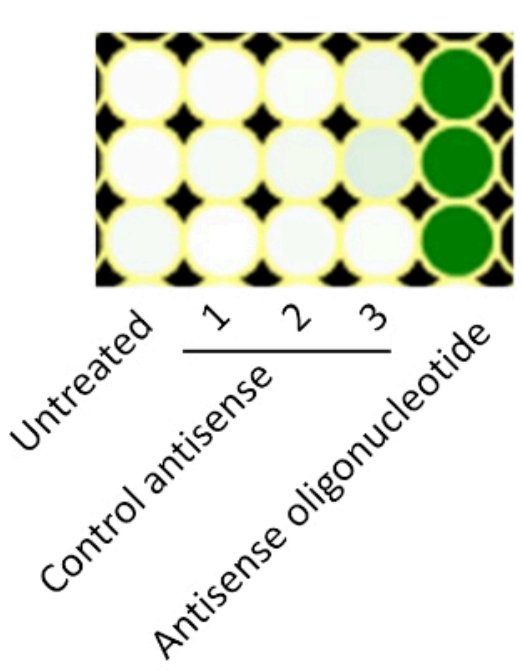

B

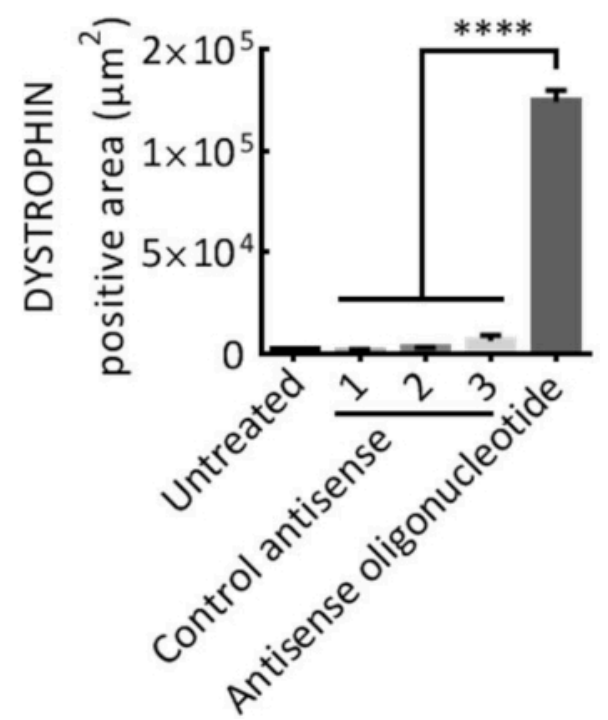

Figure 5: Heatmaps of immunocytochemistry for dystrophin after antisense oligonucleotide treatment in DZNep-treated MYOD1-UDCs obtained from DMD patient with exon 45-54 deletion. (A) Deletion of exon 45-54 restored the open reading frame based on the exon skipping of exon 44. (B) The signal intensity was quantified using a fluorescent microscope after 1 week of antisense oligonucleotide transfection on a 96 well plate. One-way ANOVA followed by Bonferroni's post hoc test was used for the comparison $\left(n=3-4\right.$ for each group, $\left.{ }^{* * * *} P<0.0001\right)$. This figure has been modified from Takizawa et al. ${ }^{7}$. Please click here to view a larger version of this figure. 


\begin{tabular}{|l|l|l|}
\hline Reagent & Volume & Final concentration \\
\hline 2x PCR premix & $12.5 \mu \mathrm{L}$ & $1 \mathrm{x}$ \\
\hline Forward primer & $5 \mathrm{pmol}$ & $0.2 \mu \mathrm{M}$ \\
\hline Reverse primer & $5 \mathrm{pmol}$ & $0.2 \mu \mathrm{M}$ \\
\hline Template & $80 \mathrm{ng}$ & \\
\hline Sterilized distilled water & up to $25 \mu \mathrm{L}$ & \\
\hline Total volume per reaction & $\mathbf{2 5} \boldsymbol{\mu L}$ & \\
\hline
\end{tabular}

Table 1: Mixture for MYOD1 amplification by RT-PCR.

\begin{tabular}{|l|l|l|}
\hline $98^{\circ} \mathrm{C}$ & $10 \mathrm{~s}$ & \\
\hline $55^{\circ} \mathrm{C}$ & $10 \mathrm{~s}$ & \} 35 cycles \\
\hline $72{ }^{\circ} \mathrm{C}$ & $10 \mathrm{~s}$ & \\
\hline
\end{tabular}

Table 2: Conditions for the thermal cycler for MYOD1 amplification.

\begin{tabular}{|l|l|}
\hline Reagent & Volume \\
\hline $10 x$ K buffer & $2 \mu \mathrm{L}$ \\
\hline Retroviral vector $(500 \mathrm{ng} / \mu \mathrm{L})$ & $2 \mu \mathrm{L}$ \\
\hline Restriction enzyme 1 (2-15 U) & $1 \mu \mathrm{L}$ \\
\hline Restriction enzyme 2 (2-15 U) & $1 \mu \mathrm{L}$ \\
\hline Sterilized distilled water & $14 \mu \mathrm{L}$ \\
\hline Total volume & $\mathbf{2 0} \boldsymbol{\mu L}$ \\
\hline
\end{tabular}

Table 3: Mixture for a tube to digest retroviral vector.

\begin{tabular}{|l|l|}
\hline Reagent & Volume \\
\hline Purified MYOD1 fragment & $100 \mathrm{ng}$ \\
\hline Digested retroviral vector & $100 \mathrm{ng}$ \\
\hline $5 x$ Enzyme premix & $4 \mu \mathrm{L}$ \\
\hline Sterilized distilled water & up to $20 \mu \mathrm{L}$ \\
\hline Total volume & $\mathbf{2 0} \mu \mathrm{L}$ \\
\hline
\end{tabular}

Table 4: Mixture for the in-fusion cloning reaction.

\begin{tabular}{|l|l|l|}
\hline Solution & Volume/Reaction $(\boldsymbol{\mu L})$ & Final concentration \\
\hline RNase-free water & Variable & - \\
\hline One-step RT-PCR buffer & 4 & $1 \mathrm{x}$ \\
\hline dNTP mix (containing $10 \mathrm{mM}$ of each dNTP) & 0.8 & $400 \mathrm{mM}$ of each dNTP \\
\hline Forward primer (10 mM) & 1.2 & $0.6 \mathrm{mM}$ \\
\hline Reverse primer (10 mM) & 1.2 & $0.6 \mathrm{mM}$ \\
\hline One-step RT-PCR enzyme mix & 0.8 & - \\
\hline RNase inhibitor (optional) & Variable & $5-10$ units/reaction \\
\hline Template RNA & $50-400 \mathrm{ng}$ & \\
\hline Total volume & $\mathbf{2 0}$ & \\
\hline
\end{tabular}

Table 5: Necessary compounds for one reaction of the one-step RT-PCR. 


\begin{tabular}{|l|l|l|l|}
\hline 1 cycle & Reverse transcription & $30 \mathrm{~min}$ & $50^{\circ} \mathrm{C}$ \\
\hline 1 cycle & Initial PCR activation step & $15 \mathrm{~min}$ & $95^{\circ} \mathrm{C}$ \\
\hline \multirow{3}{*}{1 cycle } & Denaturation & $1 \mathrm{~min}$ & $94^{\circ} \mathrm{C}$ \\
\cline { 2 - 4 } & Annealing & $1 \mathrm{~min}$ & $60^{\circ} \mathrm{C}$ \\
\cline { 2 - 4 } & Extension & $1 \mathrm{~min}$ & $72^{\circ} \mathrm{C}$ \\
\hline 1 cycle & Final extension & $7 \mathrm{~min}$ & $72^{\circ} \mathrm{C}$ \\
\hline Hold & & $\infty$ & $4^{\circ} \mathrm{C}$ \\
\hline
\end{tabular}

Table 6: Thermal cycler condition for one-step RT-PCR.

\begin{tabular}{|l|l|}
\hline Reagent & Volume \\
\hline Protein $(15 \mu \mathrm{g})$ & $10 \mu \mathrm{L}$ \\
\hline Sample buffer $(4 \mathrm{x})$ & $5 \mu \mathrm{L}$ \\
\hline Reducing agent $(10 \mathrm{x})$ & $2 \mu \mathrm{L}$ \\
\hline Deionized water & $3 \mu \mathrm{L}$ \\
\hline Total volume & $\mathbf{2 0} \mu \mathrm{L}$ \\
\hline
\end{tabular}

Table 7: Preparation of samples for sodium dodecyl sulfate polyacrylamide gel electrophoresis (SDS-PAGE).

\section{Discussion}

Here, we describe a detailed protocol of exon skipping in MYOD1-converted UDCs obtained from DMD patients. Using the assay system, we screened optimal antisense sequences efficiently. We assume that MYOD1-converted UDCs can be useful for the investigation of the pathophysiology of the disease.

Evaluation of exon skipping using patient-derived cells at the mRNA level is indispensable for screening new drugs and assessing patient eligibility before undertaking clinical trials. Calculation of exon skipping efficiency can be evaluated only at an mRNA level.

Evaluation of exon skipping at the protein level is also important because dystrophin restoration is important as a surrogate biomarker to predict the benefits of exon skipping. To date, screening of antisense oligonucleotide sequences is often performed using primary muscle cell lines or immortalized myoblast cell lines including human rhabdomyosarcoma (RD) cells, but we cannot measure the recovery of dystrophin levels using muscle cell lines or RD cell lines because they express this protein endogenously. We can clearly detect the restoration of dystrophin in DMD patient-derived MYOD1-UDCs in a dose-dependent manner. In our new assay, we consider that the evaluation of restored protein by Western blotting is superior in quantifiability. On the other hand, evaluation by immunocytochemistry using 96 well plates is ideal for screening many candidate compounds simultaneously.

In this article, we describe a detailed protocol for an efficient modelling DMD muscle using MYOD1-converted UDCs along with RT-PCR, Western blotting, and immunocytochemistry to evaluate the restored dystrophin at the mRNA and protein levels after exon skipping. UDCs can be collected noninvasively and easily. Therefore, we assume that the brand-new in vitro assay can be applied to a wide range of basic and translational studies regardless of the type of muscular disorders.

\section{Disclosures}

The National Center of Neurology and Psychiatry is now developing NS-065/NCNP-01, an exon 53 skipping drug for DMD, with Nippon Shinyaku Co., Ltd.

\section{Acknowledgments}

This work was supported by the Japan Society for the Promotion of Science Grant-in-Aid for Scientific Research (C) [grant no. 18K07544 to Y.A.], Grants-in-Aid for Research on Nervous and Mental Disorders [grant no. 28-6 to Y.A.], and the Japan Agency for Medical Research and Development [grant nos. 18ek0109239h0002, 18Im0203066h0001, and 18Im0203069h0001 to Y.A.].

\section{References}

1. Hoffman, E. P., Brown, R. H., Jr., Kunkel, L. M. Dystrophin: the protein product of the Duchenne muscular dystrophy locus. Cell. 51, 919-928 (1987).

2. Cirak, S. et al. Exon skipping and dystrophin restoration in patients with Duchenne muscular dystrophy after systemic phosphorodiamidate morpholino oligomer treatment: an open-label, phase 2, dose-escalation study. Lancet. 378, 595-605 (2011).

3. Komaki, H. et al. Systemic administration of the antisense oligonucleotide NS-065/NCNP-01 for skipping of exon 53 in patients with Duchenne muscular dystrophy. Science Translational Medicine. 10 (437), eaan0713 (2018). 
4. Antoury, L. et al. Analysis of extracellular mRNA in human urine reveals splice variant biomarkers of muscular dystrophies. Nature Communications. 9, 3906 (2018).

5. Rahmoune, $\mathrm{H}$. et al. Glucose transporters in human renal proximal tubular cells isolated from the urine of patients with non-insulin-dependent diabetes. Diabetes. 54, 3427-3434 (2005).

6. Zhang, Y. et al. Urine derived cells are a potential source for urological tissue reconstruction. The Journal of Urology. 180, 2226-2233 (2008).

7. Takizawa, H. et al. Modelling Duchenne muscular dystrophy in MYOD1-converted urine-derived cells treated with 3-deazaneplanocin A hydrochloride. Scientific Reports. 9, 3807 (2019).

8. Zhou, T. et al. Generation of human induced pluripotent stem cells from urine samples. Nature Protocols. 7, $2080-2089$ (2012).

9. Chen, W. et al. Skeletal myogenic differentiation of human urine-derived cells as a potential source for skeletal muscle regeneration. Journal of Tissue Engineering and Regenerative Medicine. 11, 334-341 (2017).

10. Kim, E. Y., Page, P., Dellefave-Castillo, L. M., McNally, E. M., Wyatt, E. J. Direct reprogramming of urine-derived cells with inducible MyoD for modeling human muscle disease. Skeletal Muscle. 6, 32 (2016). 